\title{
Zofenopril: Blood pressure control and cardio-protection
}

\author{
Claudio Borghi ${ }^{1}$, Giuseppe Ambrosio ${ }^{2}$, Philippe Van De Borne ${ }^{3}$, Giuseppe Mancia ${ }^{4}$ \\ ${ }^{1}$ Unit of Internal Medicine, Policlinico S. Orsola, University of Bologna, Italy \\ ${ }^{2}$ Division of Cardiology, University of Perugia School of Medicine, Perugia, Italy \\ ${ }^{3}$ Department of Cardiology, Erasme University Hospital, Université Libre de Bruxelles, Brussels, Belgium \\ ${ }^{4}$ University of Milano-Bicocca, Milan and Policlinico di Monza, Monza, Italy
}

\begin{abstract}
Current hypertension guidelines suggest various strategies to reduce blood pressure levels, thereby reducing cardiovascular events: combinations of drugs with different mechanisms of action, such as an angiotensin converting enzyme inhibitors (ACEIs) and a diuretic, are the cornerstone of the modern treatment of hypertension, also as initial therapy. Among ACEIs, zofenopril has been shown to be effective in the management of hypertension both as monotherapy and in combination with a diuretic: zofenopril/hydrochlorothiazide fixed dose combination is particularly useful to improve treatment adherence through simplification of treatment regimen. Moreover, thanks to the sulfhydryl group, zofenopril has some peculiar properties (higher lipophilicity and tissue penetration, lower bradykinin-dependent effect, higher affinity for, and more persistent binding to, tissue ACE, significant antioxidant effect), which may account for the cardioprotective effects of the drug demonstrated in both pre-clinical studies and randomized clinical trials. The positive impact of zofenopril on clinical outcomes has been extensively documented by the SMILE program, including several clinical trials in patients with different conditions of myocardial ischemia treated with zofenopril: the results of the SMILE program, demonstrating the benefits of zofenopril vs. placebo and other ACEIs, emphasize the importance of a differentiated approach to patients with ischemic heart disease, based on a careful choice of the adopted agent, in order to improve the overall impact of pharmacological treatment on clinical outcomes. (Cardiol J 2022; 29, 2: 305-318)
\end{abstract}

Key words: zofenopril, hypertension, coronary artery disease, cardioprotection, nitric oxide, vasodilation, sulfhydrylic angiotensin converting enzyme inhibitors, fixed dose combination

\section{Background}

Despite the substantial progress made in understanding the epidemiology, pathophysiology, and risk associated with hypertension (HTN) and the treatment strategies currently available to reduce blood pressure (BP), HTN remains a major preventable cause of cardiovascular (CV) disease (CVD) and all-cause death globally [1].

Available evidence demonstrates that BP lowering can substantially reduce premature mor- bidity and mortality. Meta-analyses of randomized clinical trials (RCTs) including several hundred thousand patients have shown that a $10-\mathrm{mmHg}$ reduction in systolic $\mathrm{BP}$ (SBP) or a $5-\mathrm{mmHg}$ reduction in diastolic BP (DBP) is associated with significant reductions in all major $\mathrm{CV}$ events by $20 \%$, all-cause mortality by $10-15 \%$, stroke by $35 \%$, coronary events by $20 \%$, and heart failure (HF) by $40 \%$. These relative risk reductions are consistent, irrespective of baseline $\mathrm{BP}$ within the hypertensive range, level of $\mathrm{CV}$ risk, comorbidities

Address for correspondence: Claudio Borghi, MD, Divisione di Medicina Interna, Policlinico S. Orsola, Via Massarenti 9, 40138 Bologna, Italy, tel: +39 051 6363243, fax: +39051391320, e-mail: claudio.borghi@unibo.it

Received: 8.04.2021 Accepted: 5.07.2021 Early publication date: 30.09.2021

This article is available in open access under Creative Common Attribution-Non-Commercial-No Derivatives 4.0 International (CC BY-NC-ND 4.0) license, allowing to download articles and share them with others as long as they credit the authors and the publisher, but without permission to change them in any way or use them commercially. 
(e.g., diabetes and chronic kidney disease [CKD]), age, sex, and ethnicity [1].

The 2018 European Society of Cardiology/ /European Society of Hypertension (ESC/ESH) hypertension guidelines have introduced many therapeutic novelties, aiming at optimizing HTN treatment and improving BP control. The most important innovations include an earlier start of pharmacologic treatment (which is now recommended in patients with high normal BP and very high CV risk, as well as in patients with grade 1 HTN and high or very high CV risk), lower BP targets $(\leq 130 / 80 \mathrm{mmHg}$ in most patients, if tolerated, regardless of $\mathrm{CV}$ risk or comorbidities), and a less conservative approach to old ( $\geq 65$ years) and very old patients ( $>80$ years), with lower treatment thresholds (grade $1 \mathrm{HTN}$ [SBP 140$-159 \mathrm{mmHg}$ ] in old fit patients), and lower BP targets $(<130-139 \mathrm{mmHg}$ for old and very old patients, if tolerated) [1].

Another major novelty is that guidelines have abandoned the recommendation of monotherapy for treatment initiation, in favor of an initial dual drug combination in most patients, in order to minimize low adherence and therapeutic inertia, known to be the major causes of low BP control in the hypertensive population. Moreover, initial combination treatment results in reduced treatment discontinuation and a lower risk of $\mathrm{CV}$ events. Single pill combinations are recommended as the best choice for initiation of dual drug pharmacologic treatment in most patients, because reducing the number of pills to be taken daily improves adherence and increases the rate of BP control [1].

New guidelines continue to consider the main 5 drug classes - i.e., angiotensin converting enzyme inhibitors (ACEIs), angiotensin receptors blockers (ARBs), calcium channel blockers (CCBs), diuretics, and beta-blockers (BBs) - as the most suitable for treatment initiation and maintenance, because for each of them there is evidence of BP-lowering effectiveness, protective effect vs. placebo and, in most trials and meta-analyses, similar degree of protection in comparison studies. There are also data from randomized trials of $\mathrm{CV}$ protection by their combined use [1].

According to new guidelines, combinations of drugs with different mechanisms of action are the cornerstone of the modern treatment of HTN. Preferred combinations should comprise a blocker of the renin-angiotensin-aldosterone system (RAAS), with a CCB or a diuretic; these combinations have the advantage of being complementary, because both CCBs or diuretics activate the RAAS, which will be counteracted by their combination with a RAAS blocker. These combinations will also limit potential adverse effects associated with diuretic or CCB monotherapy, reducing the risk of hypokalemia due to diuretics and the prevalence of peripheral edema due to CCBs [1].

In the context of the newly recommended main treatment strategy, guidelines have upgraded the use of an RAAS blocker with a CCB or diuretic not only because of the evidence of their protective effect in uncomplicated HTN or in the presence of HTN-mediated organ damage (HMOD) or specific comorbidities, but also for their greater availability in single pill combinations [1].

The pharmacological blockade of the RAAS can be achieved with either inhibition of the angiotensin converting enzyme (with ACEIs) or blockade of the angiotensin receptor (with ARBs): both classes are strongly recommended, because beyond their anti-hypertensive action, they reduce albuminuria more than other BP-lowering drugs and are effective at delaying the progression of diabetic and non-diabetic CKD. ACEIs and ARBs also appear to be effective in preventing or regressing HMOD, such as left ventricular hypertrophy and small artery remodeling, for an equivalent reduction in BP. Both drugs reduce incident atrial fibrillation $(\mathrm{AF})$, which may be related to improved left ventricular (LV) function and more effective LV structural regression. Due to the huge body of trial-based evidence, ACEIs and ARBs are the pillar of antihypertensive therapy, playing a major role in both primary and secondary CV prevention [1].

The purpose of the present review is to summarize the current evidence supporting the effectiveness of ACEIs in terms of BP control and CV protection, with a focus on sulfhydrylic ACEIs in general, and zofenopril in particular, highlighting the peculiar pharmacological properties underlying its favorable efficacy profile.

\section{The key role of ACEIs in HTN and $\mathrm{CV}$ protection}

Given the pivotal role of angiotensin II in the pathogenesis of CVD, ACEIs play a key role in HTN treatment: by blocking the conversion of angiotensin I to angiotensin II, they exert reno- and cardio-protective effects in addition to anti-hypertensive activity, which makes them an excellent option for front-line management of HTN in patients with associated risk factors. Reduced BP with ACEI monotherapy is reported in 35-70\% of patients, whereas response rates $>80 \%$ in 
patients with mild to moderate HTN treated with an $\mathrm{ACEI} /$ diuretic combination have been reported. Moreover, this class of agents is associated with few metabolic adverse effects, both in monotherapy and in combination [2].

Thus, ACEIs have an unchallenged position among available antihypertensive drugs as first-step treatment of HTN: they are effective for uncomplicated HTN and asymptomatic HMOD [1], and are the preferred drugs for the treatment of many specific conditions ( $\mathrm{AF} / \mathrm{HF} /$ post-stroke/ /post-myocardial infarction/peripheral artery disease/CKD) [3].

Activation of RAAS has long been implicated also in the pathogenesis of acute myocardial infarction (AMI), and its blockade through use of ACEIs has been shown to be beneficial in preventing major $\mathrm{CV}$ complications in several large, randomized, prospective, early and late intervention trials in patients with post-AMI[4].

Based on this evidence, ACEIs have been recognized as holding a key position in the primary antihypertensive treatment strategy, as a part of initial combination therapy [1], as well as in the management of AMI, as an essential component of treatment of patients with ST segment elevation anterior AMI, post-AMI LV dysfunction (LV ejection fraction $[\mathrm{LVEF}]<40 \%$ ), or those who have experienced HF in the early phase of AMI $[5,6]$.

\section{Pharmacological properties of sulfhydrylic ACEIs: Which advantages for cardio-protection?}

After the discovery of captopril, the prototype of orally active ACEIs, several new ACEIs were developed and introduced into medical practice, differing in their chemical structure, functional groups (sulfhydryl in captopril and zofenopril, carboxyl in enalapril, phosphinyl in fosinopril), active moiety (some are prodrugs), potency, ancillary pharmacological actions, and pharmacokinetics [7].

Sulfhydrylic ACEIs have some peculiar aspects, contributing to their pharmacological properties: i) higher lipophilicity and tissue penetration; ii) lower bradykinin-dependent effect; iii) higher affinity and more persistent binding to tissue ACE (cardiac/renal/vascular); and iv) significant antioxidant effect [7].

The most recent sulfhydrylic ACEI to reach the European market was zofenopril, a highly lipophilic drug, characterized by increased oral absorption, an appreciable degree of biliary excretion, and enhanced tissue penetration [7].
Zofenopril is unique in producing long-lasting and selective inhibition of heart tissue ACE, which is likely determined by the high efficiency with which the prodrug is taken up by heart tissue and promptly hydrolyzed by cardiac esterases to the active inhibitor carrying a sulfhydryl group, zofenoprilat [7], which is 6-10 times more potent than captopril, displaying an $\mathrm{EC}_{50}$ in the nanomolar range (1-8 nM) [8]. Compared to captopril, zofenopril produces a dose-dependent antihypertensive effect of longer duration ( $>17 \mathrm{~h}$ ) in animal models of hypertension [7].

The presence of an sulfhydrylic group grants peculiar cardio-protective properties to sulfhydrylic ACEIs: in vitro studies in experimental models of ischemia/reperfusion (I/R) demonstrated the cardio-protective properties of zofenopril, in terms of improvement of post-ischemic LV function (LVF), increased coronary flow, and reduced creatine kinase release, as well as reduced lipid peroxidation during reperfusion. Although these cardio-protective effects were observed with both zofenopril and captopril, zofenopril was more potent. In particular, in isolated perfused hearts, zofenopril $10^{-5} \mathrm{M}$ exerted a greater effect than captopril in restoring cardiac function after $\mathrm{I} / \mathrm{R}[9,10]$.

In vivo animal studies confirmed the ability of zofenopril to prevent ischemic myocardial damage, as well as to reduce post-ischemic cardiac remodeling by suppressing the increase of both ventricular mass and volume [7].

The potential of sulfhydrylic ACEIs in scavenging radical oxygen species has been proposed as a co-causative factor in the cardio-protection exerted by this class of compounds [7]. This may be due to the fact that the sulfhydrylic group is a potent proton donor, exerting an important anti-oxidant activity; in this regard, the cardioprotective effect of zofenopril in murine and swine models of myocardial I/R injury was associated with an increase in hydrogen sulfide $\left(\mathrm{H}_{2} \mathrm{~S}\right)$ bioavailability [10]. An additional mechanism by which $\mathrm{H}_{2} \mathrm{~S}$ confers cardio-protection against I/R injury and pressure-overload HF is through its ability to enhance endothelial nitric oxide synthase (eNOS) activity and thereby increase myocardial nitric oxide (NO) bioavailability [10]. In vivo studies demonstrated that the modulation of $\mathrm{H}_{2} \mathrm{~S}$ by zofenopril represents an additional beneficial mechanism unrelated to ACE-inhibition [11]. Experimental data suggest therefore that zofenopril may provide a double advantage, deriving from both ACE-inhibition and increased $\mathrm{H}_{2} \mathrm{~S}$ bioavailability (Fig. 1) [10]: by inhibiting myocardial ACE activity, zofenopril reduces 


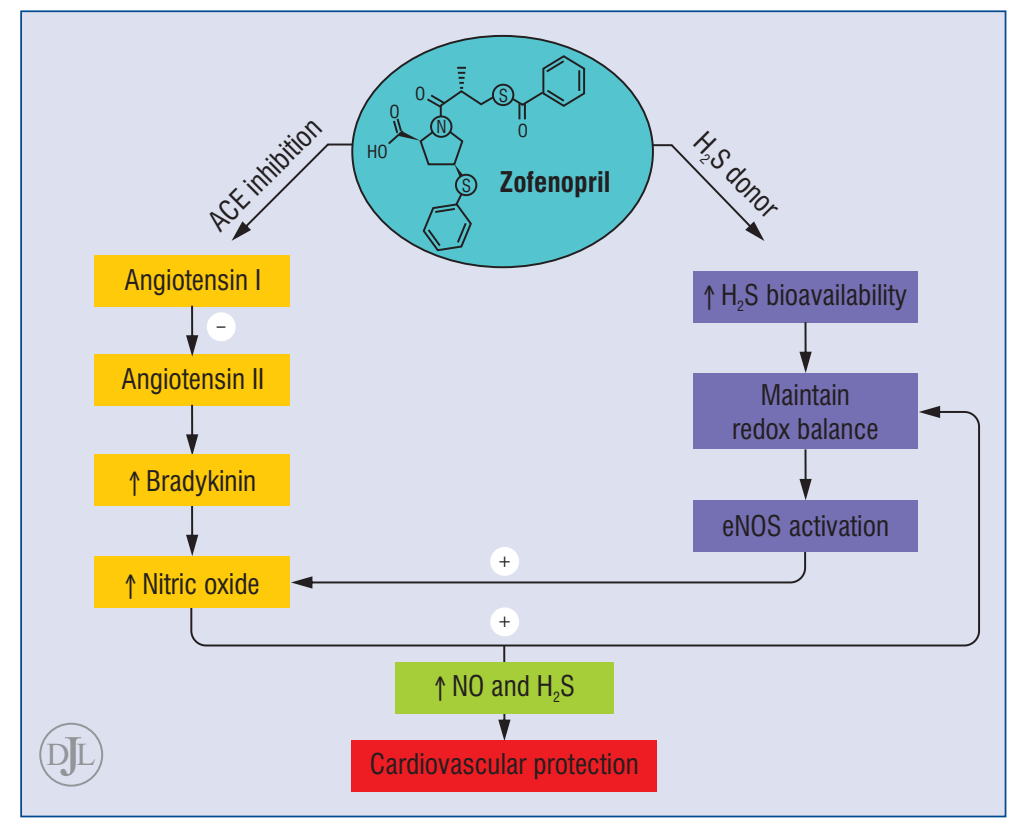

Figure 1. Effect of zofenopril on hydrogen sulfide $\left(\mathrm{H}_{2} \mathrm{~S}\right)$ and nitric oxide (NO) bioavailability (reproduced from [10]); ACE - angiotensin converting enzyme; eNOS - endothelial nitric oxide synthase.

the conversion of angiotensin I to angiotensin II and increases the levels of bradykinin. Bradykinin, through stimulation of endothelial $\mathrm{B}_{2}$ receptors, promotes the release of $\mathrm{NO}$, prostacyclin, and endothelium-derived hyperpolarizing factor (EDHF), as well as eNOS activation, leading to increased levels of NO [10].

The increased NO availability induced by the activation of both these pathways may account for the greater benefits seen with zofenopril compared with other ACEIs; this has been confirmed in vitro by measuring the stimulation of NO release by endothelial cells induced by captopril, enalapril, or zofenopril, the latter being much more effective than the comparators [12]. Similarly, zofenoprilat was shown to be more effective than captopril, lisinopril, and enalaprilat in reducing endothelin-1 secretion from cultured human vascular endothelial cells (HUVECs), an effect most likely due to the sulfhydryl-related scavenging capability and the consequent decrease in NO inactivation by endogenous oxidants [13].

The beneficial effect of NO in terms of cardioprotection might also depend upon its role in ischemic preconditioning, a phenomenon by which a prolonged episode of ischemia paradoxically induces much less injury than expected if it occurs after a brief "preconditioning" episode of ischemia: indeed, it has been demonstrated that new-onset angina preceding AMI is associated with lower release of markers of cell necrosis, and improved contractile recovery after thrombolysis [14].

Consistent with the role of NO in inducing preconditioning, pharmacological preconditioning may be achieved through NO donors by using chronic nitrate therapy, as documented by the results of the Global Registry of Acute Coronary Events: in this large multinational registry, including 52,693 patients from 123 centers in 14 countries, chronic nitrate use was associated with a shift away from ST segment elevation MI in favor of non-ST segment elevation MI-acute coronary syndrome, and with less release of markers of cardiac necrosis. These findings suggest that in chronic nitrate users, acute coronary events may develop to a lesser extent [15].

It is now recognized that $\mathrm{NO}$ exposure before ischemia may act as a potent preconditioning mimetic and cardio-protectant, and it may represent the basis of potential infarct-sparing strategies; activation of NO synthase or production of NO can be obtained pharmacologically with exogenous agents able to trigger this cascade [16], such as the sulfhydryl-containing ACEI zofenopril.

\section{Clinical efficacy of zofenopril in complicated patients: The SMILE project}

The positive impact of the cardio-protective effect of zofenopril on clinical outcomes has been 
Table 1. The Survival of Myocardial Infarction Long-term Evaluation (SMILE) program.

\begin{tabular}{lc}
\hline Study [reference] & Main features \\
\hline SMILE Pilot Study [17] & 204 non-thrombolyzed patients \\
Zofenopril vs. standard treatment & Safety assessment \\
\hline SMILE Study [18] & 1556 non-thrombolyzed patients \\
Zofenopril vs. placebo & 6-week incidence of death or severe CHF \\
& 1-year mortality rate \\
\hline SMILE-2 Study [19] & 1024 thrombolyzed patients \\
& Zofenopril vs. lisinopril \\
& 6-week rate of severe hypotension \\
& 6-week safety profile \\
\hline SMILE-3 Ischemia & 349 thrombolyzed patients with preserved LV EF \\
Study [20] & Zofenopril vs. placebo \\
& 6-month global ischemic burden \\
\hline SMILE-4 Study [21] & 771 patients with systolic LV dysfunction (EF < 45\%) \\
Zofenopril + ASA vs. ramipril + ASA
\end{tabular}

ASA — acetylsalicylic acid; CHF — congestive heart failure; CV — cardiovascular; EF — ejection fraction; LV — left ventricular

extensively documented by the SMILE project, including several clinical trials in patients with different conditions of myocardial ischemia treated with zofenopril (Table 1) [17-21].

The 5 double-blind, randomized, parallel-group SMILE studies [17-21] compared the efficacy and safety of zofenopril with that of placebo (SMILE Pilot [17], SMILE-1 [18] and SMILE-3 [20]), lisinopril (SMILE-2) [19], or ramipril (SMILE-4) [21] in European men and non-pregnant women with AMI. Patients included in the studies were those with the following: (1) an early $\operatorname{AMI}(<24 \mathrm{~h})$, not eligible for thrombolytic therapy because of late admission to the intensive care unit or with contraindication to systemic fibrinolysis (SMILE-1) [18]; (2) a confirmed diagnosis of AMI and a prior thrombolytic treatment within $12 \mathrm{~h}$ of the onset of clinical symptoms of AMI (SMILE-2) [19]; (3) a recent AMI (within $6 \pm 1$ weeks) with preserved LVEF ( $>40 \%$ ), treated with a thrombolytic treatment and with ACEIs (SMILE-3) [20]; and (4) an early MI ( $<24 \mathrm{~h})$, either treated with thrombolysis or not, with primary percutaneous transluminal angioplasty or coronary artery by-pass graft, and with clinical and/or echocardiographic evidence of LV dysfunction (LVD) (SMILE-4) [21].

The antioxidant properties of zofenopril and its ability to modulate the availability of $\mathrm{NO}$ and the vascular tone at the coronary level may be responsible for the anti-ischemic effect of the drug originally described in the SMILE Pilot Trial, in which the outcome of post-MI patients was significantly improved by the treatment with zofenopril compared to placebo [17]. In-hospital and longterm consequences of treatment with zofenopril initiated within $24 \mathrm{~h}$ from the onset of symptoms were compared with those of standard treatment in this open-label trial involving 204 patients with AMI not undergoing thrombolytic treatment. The in-hospital incidence of acute LV failure and ventricular arrhythmias decreased by $63 \%$ and $39 \%$, respectively, among zofenopril-treated patients, who also reported fewer angina episodes both acutely (68\% reduction) and over the long term (56\% reduction). LV size decreased and LVEF increased in patients who received zofenopril, and the improvement was greater among patients with poorer ventricular function $(\mathrm{EF}<40 \%)$. These results suggest that early administration of zofenopril may be effective in patients with AMI, particularly when the event is complicated by clinical signs or evidence of LVD [17].

The benefits of zofenopril for patients with AMI and LVD were confirmed by the SMILE-1 study, in which zofenopril was able to improve prognosis during the very early phase (i.e., within $48 \mathrm{~h}$ from the onset of symptoms) and the mid phase of the disease (i.e., after 6 weeks of double-blind 
treatment), as well as over 1 year of treatment [18]. Among 1556 enrolled patients within $24 \mathrm{~h}$ of the onset of symptoms of anterior AMI and randomly assigned in a double-blind fashion to receive either placebo (784 patients) or zofenopril (772 patients) for 6 weeks, the cumulative reduction in the risk of death or severe congestive $\mathrm{HF}$ was $34 \%$ at 6 weeks ( $46 \%$ for severe congestive $\mathrm{HF}$ and $25 \%$ for death). The beneficial effect of short-term treatment with zofenopril was maintained over time: after 1 year of observation, the mortality rate was significantly lower in the zofenopril group than in the placebo group $(10.0 \%$ vs. $14.1 \%, 29 \%$ risk reduction; $\mathrm{p}=0.011$ ). These results confirmed the benefits of early treatment with zofenopril, significantly improving both short-term and long-term outcomes when started within $24 \mathrm{~h}$ of onset of anterior AMI and continued for 6 weeks [18].

The SMILE-2 study, a phase III, double-blind, parallel-group, multicenter study comparing the safety and efficacy of zofenopril (30-60 mg/day) and lisinopril (5-10 mg/day), starting within $12 \mathrm{~h}$ of completion of thrombolytic therapy and continuing for 42 days, in 1024 thrombolyzed patients with AMI, demonstrated that both zofenopril and lisinopril are safe and associated with a rather low rate of severe hypotension when given in accordance with a dose-titrated scheme. The incidence of drug-related severe hypotension was slightly but significantly lower with zofenopril than with lisinopril (6.7\% vs. $9.8 \%, 2$-tailed $p=0.048)$. These findings could have a positive clinical impact and increase the proportion of patients with AMI who can be safely treated with ACEIs [19].

The cardio-protective role of zofenopril when given to patients with normal LVF after AMI were confirmed by the SMILE-3 Ischemia Study, which demonstrated a significant reduction of the ischemic burden (defined as significant ST-T abnormalities on ambulatory electrocardiography, electrocardiography abnormalities or symptoms of angina during standard exercise test, recurrence of MI, and need for revascularization procedures for angina) in 349 post-MI patients with preserved LVF (LVEF > 40\%) treated for 6 months with zofenopril 30 to $60 \mathrm{mg}(\mathrm{n}=177)$ or placebo $(\mathrm{n}=172)$ according to a double-blind, randomized study design [20]. The primary endpoint (global ischemic burden) occurred in $20.3 \%$ of zofenopril-treated and $35.9 \%$ of placebo-treated patients (44\% risk reduction, $\mathrm{p}=0.001$ ), despite no differences in $\mathrm{BP}$ control, LVF, and concomitant therapy. The rate of major $\mathrm{CV}$ events was reduced in patients treated with zofenopril, with a lower rate of development and progression of congestive HF. The results of the SMILE Ischemia study extend the benefits of zofenopril in terms of cardio-protection and prevention of coronary events from the early to the late phase of MI [20].

The difference between zofenopril and other ACEIs in post-MI patients complicated with LVD (clinical signs of $\mathrm{HF}$ or $\mathrm{EF}<45 \%$ ) was investigated by the SMILE-4 study, showing a higher cardioprotective effect of zofenopril compared to ramipril (both in combination with acetylsalicylic acid [ASA]). The 1-year combined occurrence of death or hospitalization for $\mathrm{CV}$ causes was significantly reduced by zofenopril vs. ramipril (odds ratio [OR]: 0.70; $\mathrm{p}=0.028$ ), as a result of a decrease in CV hospitalization (OR: 0.64; $\mathrm{p}=0.06$ ) [21]. Furthermore, the results of the SMILE-4 trial showed that differences in clinical efficacy may exist when different ACEIs are combined with ASA, demonstrating a more favorable impact of zofenopril than ramipril on major CV events and in a relatively long-term period of 1 year [21].

A retrospective analysis of the SMILE-4 study confirmed the good efficacy of zofenopril and ASA in the prevention of long-term CV outcomes also in the subgroup of patients with HTN ( $n=525$, $77 \%$ ), in which major $\mathrm{CV}$ outcomes were reported in $31 \%$ of zofenopril-treated patients and in $39 \%$ of ramipril-treated patients, with a $31 \%$ significantly $(\mathrm{p}=0.041)$ lower risk with zofenopril. The superiority of zofenopril vs. ramipril was particularly evident in patients with isolated systolic hypertension (ISH) [0.48; $p=0.045]$. The peculiar pharmacological characteristics of zofenopril, with its high efficacy on the remodeling process and on endothelial dysfunction shown in animal and human models, might explain its superior clinically efficacy in respect to ramipril [22].

The benefits of zofenopril treatment were maintained in the long term, as demonstrated by the results of the follow-up of the SMILE-4 study, in which zofenopril was superior compared to ramipril in terms of reduction of the combined endpoint of death and hospitalization (primary endpoint) in patients enrolled in the trial for more than 5 years: the primary endpoint occurred in $27.8 \%$ of patients originally randomized and treated with zofenopril and in $43.8 \%$ of patients treated with ramipril (OR: $0.65 ; p=0.041$ ), demonstrating that benefits of early treatment of patients with LVD after AMI with zofenopril are sustained over many years as compared to ramipril [23].

The favorable effects of zofenopril treatment in patients with IHD were confirmed by the results of the pooled data analysis of the SMILE studies. 


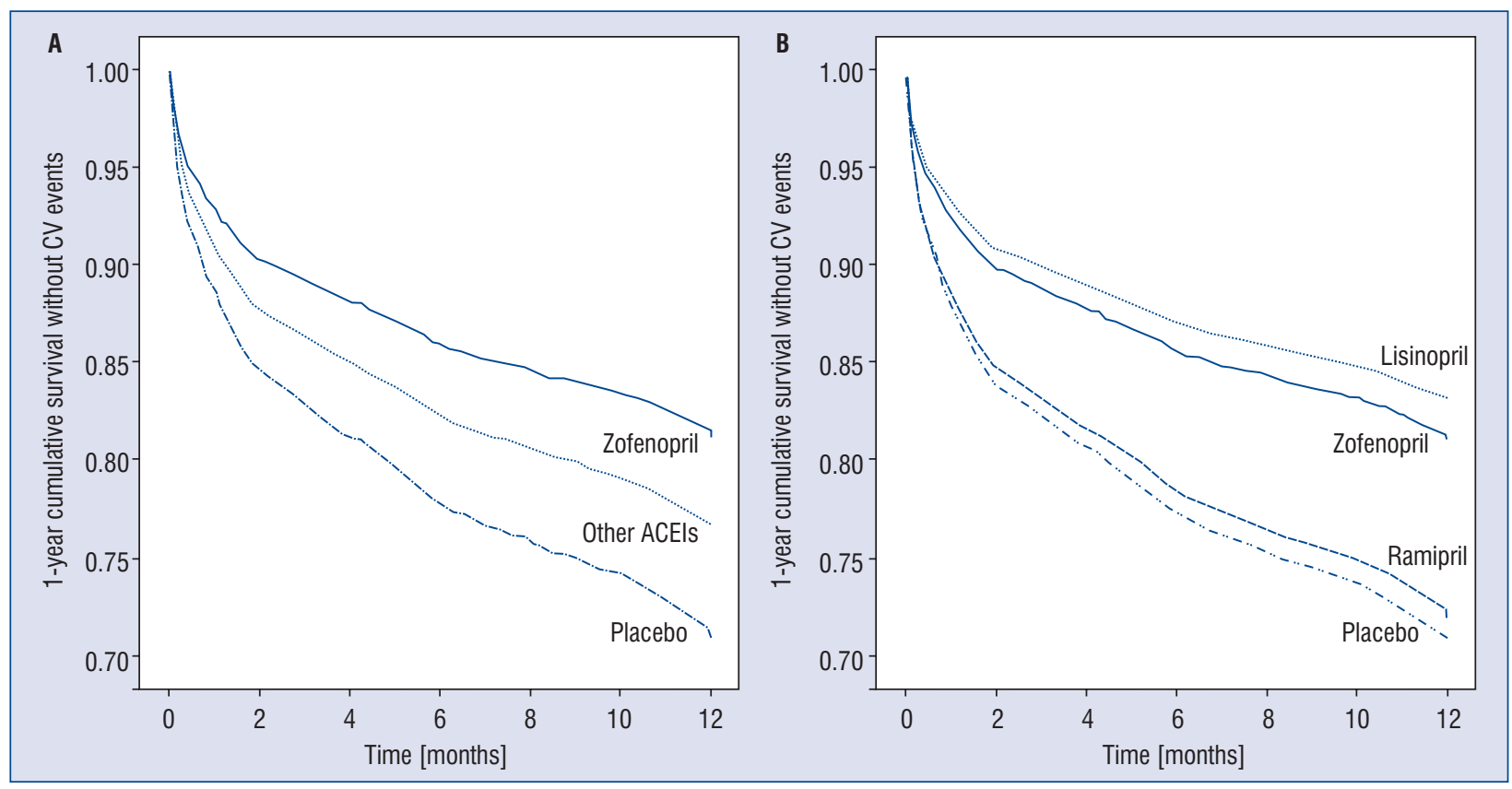

Figure 2. Cumulative survival without events during the first 42 days of treatment with zofenopril $(n=1808)$, placebo $(n=951)$, lisinopril $(n=520)$ or ramipril $(n=351)$ in the Survival of Myocardial Infarction Long-term Evaluation (SMILE) program. Data are shown by pooling together data obtained under lisinopril and ramipril (other angiotensin converting enzyme inhibitors, A) and separately for each treatment group (B) (reproduced from [4]); CV - cardiovascular.

The reduction in mortality and morbidity observed in zofenopril-treated patients in comparison to placebo and other ACEIs (Fig. 2) supports the fact that additional actions of zofenopril beyond ACE inhibition per se contribute to its efficacy in IHD [4]. These results confirm the favorable effects of zofenopril treatment in patients post AMI and its long-term benefit in terms of prevention of $\mathrm{CV}$ morbidity and mortality [4].

The results of the SMILE program emphasize the importance of a personalized approach to patients with IHD, based on careful choice of the RAAS blocking agent, in order to improve the overall impact of pharmacological treatment on clinical outcomes.

\section{Zofenopril/hydrochlorothiazide: Rationale and clinical profile}

One of the most effective 2-drug antihypertensive combinations is that combining an ACEI and a thiazide diuretic, which involves a synergistic and opposite effect on the RAAS: the thiazide diuretic reduces plasma volume with a consequent increase in plasma renin activity, aldosterone secretion, and urinary potassium loss; the ACEI, blocking the RAAS, counteracts the activity triggered by the diuretic, improving the efficacy and tolerability of single drug components [24].

The synergistic and contrasting effects on the RAAS resulting from the concomitant administration of zofenopril and hydrochlorothiazide (HCTZ) may differ from those resulting from the combination of HCTZ with other ACEIs, depending on the lipophilicity of the ACEI: HCTZ could increase the tissue concentrations of zofenopril and induce a peculiar enhancement of the tissue activity of this lipophilic ACEI, which could contribute to its protective effects [24].

Zofenopril/HCTZ combination therapy was shown to be effective in the treatment of HTN and to be superior to monotherapy in reducing office and ambulatory BP in clinical studies [24]. Zofenopril/HCTZ was also shown to be more effective than the individual compounds as monotherapy in reducing $\mathrm{BP}$ over the $24 \mathrm{~h}$, as confirmed by the higher value of smoothness index, a measure of the homogeneity of the BP control over $24 \mathrm{~h}$, observed with zofenopril 30 or $60 \mathrm{mg}$ plus HCTZ [25].

In addition, the combination of zofenopril/ /HCTZ was shown to provide more responders to treatment ( $\mathrm{DBP}$ reduction $\geq 10 \mathrm{mmHg}$ ), as well as higher normalization rates (seated DBP $<90 \mathrm{mmHg}$ ) than monotherapy [26]. 
Table 2. The $Z$ studies.

\begin{tabular}{lc}
\hline Study [reference] & Main features \\
\hline ZODIAC [29] & 361 patients with uncontrolled BP \\
& $\begin{array}{c}\text { Zofenopril + HCTZ vs. irbesartan + HCTZ } \\
\text { Office diastolic BP changes after } 18 \text { weeks }\end{array}$ \\
\hline ZENITH [30] & 462 patients with uncontrolled BP \\
& Zofenopril + HCTZ vs. irbesartan + HCTZ \\
& Office diastolic BP response after 18 weeks \\
\hline ZAMES [31] & 482 patients with uncontrolled BP \\
& Zofenopril + HCTZ vs. irbesartan + HCTZ \\
& Office diastolic BP changes after 24 weeks \\
\hline ZEUS [32] & 230 patients with uncontrolled isolated systolic hypertension \\
Zofenopril + HCTZ vs. irbesartan + HCTZ & Mean daytime systolic BP changes after 6 weeks \\
\hline
\end{tabular}

BP — blood pressure; HCTZ - hydrochlorothiazide

In addition, the fixed combination of zofenopril $30 \mathrm{mg}$ plus HCTZ $12.5 \mathrm{mg}$ resulted in improved efficacy compared with zofenopril $30 \mathrm{mg}$ alone in patients with metabolic syndrome, in whom BP control is more difficult to achieve and who are at greater risk for CV events [27]. Similar results were obtained in other high-risk conditions, such as impaired fasting glucose or diabetes, renal impairment, or dyslipidemia [28].

Although zofenopril/HCTZ was proven to be effective in lowering $\mathrm{BP}$ in patients at all quartiles of $\mathrm{CV}$ risk, it provided greater reduction of 10 -year risk of CVD in subjects at higher risk quartiles [28]. These data suggest the usefulness of this fixed combination in the treatment of patients with HTN requiring more prompt, intensive, and sustained $\mathrm{BP}$ reduction, according to guideline recommendations [28].

\section{Fixed dose combination: Z-studies}

Another effective 2-drug antihypertensive combination is that of an ARB and a thiazide diuretic, in which the ARB antagonizes the counter-regulatory system activity triggered by the diuretic [29].

Irbesartan, an ARB characterized by a high bioavailability, a long duration of action, and a small potential for pharmacological interactions, has shown a high efficacy in lowering BP in hypertensive patients, particularly those with renal impairment, showing equal efficacy but better tolerability, compared to the other major antihypertensive classes [29].

Both zofenopril and irbesartan have been successfully employed in hypertensive patients in combination with a diuretic - the efficacy of zofenopril 30 or $60 \mathrm{mg}$ and irbesartan 150 or $300 \mathrm{mg}$, both used in combination with HCTZ $12.5 \mathrm{mg}$, was directly compared in the $Z$-studies (ZODIAC, ZENITH, ZAMES, ZEUS) (Table 2) [29-32].

The primary efficacy criteria were, for all studies, the effects of the drugs on office BP; in the ZEUS study, the effect on ambulatory BP was also assessed (through ambulatory BP monitoring [ABPM]). In 2 studies, ZODIAC and ZENITH, the long-term effects of the drugs were also evaluated [33].

In the ZODIAC study, an international, multicenter, randomized, double-blind, parallel group study, conducted at 27 hospitals in 5 different European countries, 361 treated hypertensive patients were randomized to 18 -week treatment with zofenopril $30 \mathrm{mg}+\mathrm{HCTZ} 12.5 \mathrm{mg}$ or irbesartan $150 \mathrm{mg}+\mathrm{HCTZ} 12.5 \mathrm{mg}$ once daily for 18 weeks [29]. Both zofenopril + HCTZ and irbesartan + + HCTZ had similar effects in terms of office DBP/ /SBP reductions (17.6/21.5 $\mathrm{mmHg}$ vs. 15.1/20.6 $\mathrm{mmHg} ; \mathrm{p}=0.134$ for DBP and $\mathrm{p}=0.691$ for SBP), sitting office $\mathrm{BP}$ normalization $(\mathrm{SBP}<140 \mathrm{mmHg}$ and $\mathrm{DBP}<90 \mathrm{mmHg}, 79.6$ vs. $79.5 \%$; $\mathrm{p}=0.973$ ), and $24-\mathrm{h} \mathrm{DBP} / \mathrm{SBP}$ reductions $(15.4 / 21.2 \mathrm{mmHg}$ vs. $16.8 / 23.2 \mathrm{mmHg} ; \mathrm{p}=0.397$ for $\mathrm{DBP}$ and $\mathrm{p}=$ $=0.458$ for SBP). At the end of the study, serum levels of high sensitivity C-reactive protein (an indirect marker of vascular inflammation) were reduced in patients treated with zofenopril $(-0.52$ $\mathrm{mg} / \mathrm{L}$ ) and increased in those receiving irbesartan $(0.97 \mathrm{mg} / \mathrm{L})$, with a significant difference in favor of zofenopril $(\mathrm{p}=0.001)$. The results of the ZODIAC study suggest that, in previously monotherapy- 
treated, uncontrolled patients with HTN, zofenopril $30-60 \mathrm{mg}+\mathrm{HCTZ} 12.5 \mathrm{mg}$ is as effective as irbesartan 150-300 mg + HCTZ $12.5 \mathrm{mg}$, with the added value of a potential protective effect against vascular inflammation [29].

The ZENITH study, a randomized, double-blind, controlled, parallel group study conducted in 434 essential hypertensives with additional CV risk factors and uncontrolled by a previous monotherapy, treated for 18 weeks with HCTZ $12.5 \mathrm{mg}$ + zofenopril 30 or $60 \mathrm{mg}$ or irbesartan 150 or 300 $\mathrm{mg}$, specifically looked at the effect of treatment on target organ damage [30]. The antihypertensive effect was similar with the two combinations with no difference in the rate of responders in terms of both 24-h (zofenopril: $85 \%$ vs. irbesartan: $84 \%$; $\mathrm{p}=0.781$ ) and office BP (zofenopril: $68 \%$ vs. irbesartan: $70 \% ; p=0.778)$. The proportion of patients who had regression of target organ damage was similar with both drugs for cardiac damage, assessed by LV mass index, and renal damage, evaluated by albumin/creatinine ratio or microalbuminuria. The percentage of patients showing regression of vascular damage, evaluated by carotid intima/media thickness, was larger with zofenopril than with irbesartan $(31.6 \%$ vs. $16.1 \%$ reduction, respectively; $\mathrm{p}=0.0547$ ). No difference between the 2 drugs was observed in the long term: at the end of the 30 -week double-blind extension period no differences were observed in office BP response and office and 24-h BP reductions between the two treatment groups. Similarly, the long-term impact of treatment on target organ damage did not significantly differ between the two study drugs. The results of the ZENITH study demonstrated that the combination of zofenopril or irbesartan and HCTZ provide similarly effective and well tolerated control of $\mathrm{BP}$ in hypertensive patients not responding to a previous monotherapy and with a high or very high $\mathrm{CV}$ risk profile, effectively delaying the progression of CV, renal, and vascular damage of HTN [30].

The ZAMES study, a multicenter, international, randomized, double-blind, parallel group, phase III study, conducted in 482 patients randomly allocated to a fixed-dose combination of zofenopril $30 \mathrm{mg}+$ HCTZ $12.5 \mathrm{mg}$ or irbesartan $150 \mathrm{mg}+$ + HCTZ $12.5 \mathrm{mg}$ once daily for a cumulative period of 24 weeks, specifically evaluated whether treatment with the fixed-dose combination containing a sulfhydryl ACEI is at least as effective as that containing an ARB in patients with metabolic syndrome and essential HTN, uncontrolled by previous monotherapy [31]. Baseline-adjusted DBP reductions were superimposable $(\mathrm{p}=0.370)$ with zofenopril + HCTZ $(\mathrm{n}=231 ; 9.8 \mathrm{mmHg})$ and irbesartan + HCTZ $(\mathrm{n}=235 ; 10.4 \mathrm{mmHg})$. The same was for SBP (17.0 mmHg zofenopril + HCTZ vs. $18.8 \mathrm{mmHg}$ irbesartan $+\mathrm{HCTZ}, \mathrm{p}=0.113)$. The rate of normalized and responder patients $(\mathrm{SBP} / \mathrm{DBP}<140 / 90 \mathrm{mmHg}$ or SBP reduction $>20 \mathrm{mmHg}$ or DBP reduction $>10 \mathrm{mmHg}$ ) did not differ at study end $(65.8 \%$ and $77.5 \%$ zofenopril $+\mathrm{HCTZ}$ vs. $67.7 \%$ and $81.5 \%$ irbesartan + + HCTZ; $p=0.695, p=0.301)$. These results were confirmed in the 69 participants undergoing ABPM, with a comparable 24 -h average $\mathrm{BP}$ reduction (BP difference between treatment: SBP: $0.1 \mathrm{mmHg}$, $\mathrm{p}=0.975$; DBP: $-0.9 \mathrm{mmHg}, \mathrm{p}=0.541$ ). Both drugs determined similar BP reductions also in the last $6 \mathrm{~h}$ of the dosing interval [31]. There were no differences in the effects of either combination on lipid profile or fasting blood glucose, whereas the reduction of the albumin/creatinine ratio was larger for irbesartan, suggesting a slight but significantly larger effect on renal function [31]. Thus, based on the results of the ZAMES study, the combination between zofenopril and HCTZ and that of irbesartan and HCTZ both provide similarly effective, prolonged, and well tolerated control of BP in high-risk hypertensive patients with metabolic syndrome [31].

The ZEUS study, an international, multicenter, randomized, double-blind, parallel-group, phase III study, conducted in 230 patients enrolled at 24 hospitals located in 3 different European countries, randomized to 18 -week treatment with zofenopril + HCTZ $(30+12.5 \mathrm{mg}$, to be up-titrated to $60 \mathrm{mg}$ in uncontrolled patients after 6 or 12 weeks) or irbesartan + HCTZ $(150+12.5 \mathrm{mg}$, to be uptitrated to $300 \mathrm{mg}$ in uncontrolled patients after 6 or 12 weeks), specifically looked at the effect of treatment in elderly ( $>65$ years) patients with ISH, untreated or uncontrolled by a previous monotherapy [32]. The effect on BP was the same for the 2 drugs: daytime SBP reductions after 6 weeks (primary study end point) were similar with zofenopril + HCTZ and irbesartan + HCTZ (7.7 vs. $7.9, p=0.888$ ). Daytime SBP reductions were sustained during the study and were greater with low-dose zofenopril $+\mathrm{HCTZ}$ at study end (16.2 vs. $11.2 \mathrm{mmHg}, \mathrm{p}=0.028)$. The daytime SBP normalization $(<135 \mathrm{mmHg}$ ) rate was similar under zofenopril $+\mathrm{HCTZ}$ and irbesartan + $+\mathrm{HCTZ}$ at 6 and 12 weeks, but more common under zofenopril $+\mathrm{HCTZ}$ at 18 weeks $(68.2 \%$ vs. $56.0 \%, \mathrm{p}=0.031$ ). Both drugs equally reduced SBP in the last $6 \mathrm{~h}$ of the dosing interval and homogeneously reduced SBP throughout the $24 \mathrm{~h}$. The 


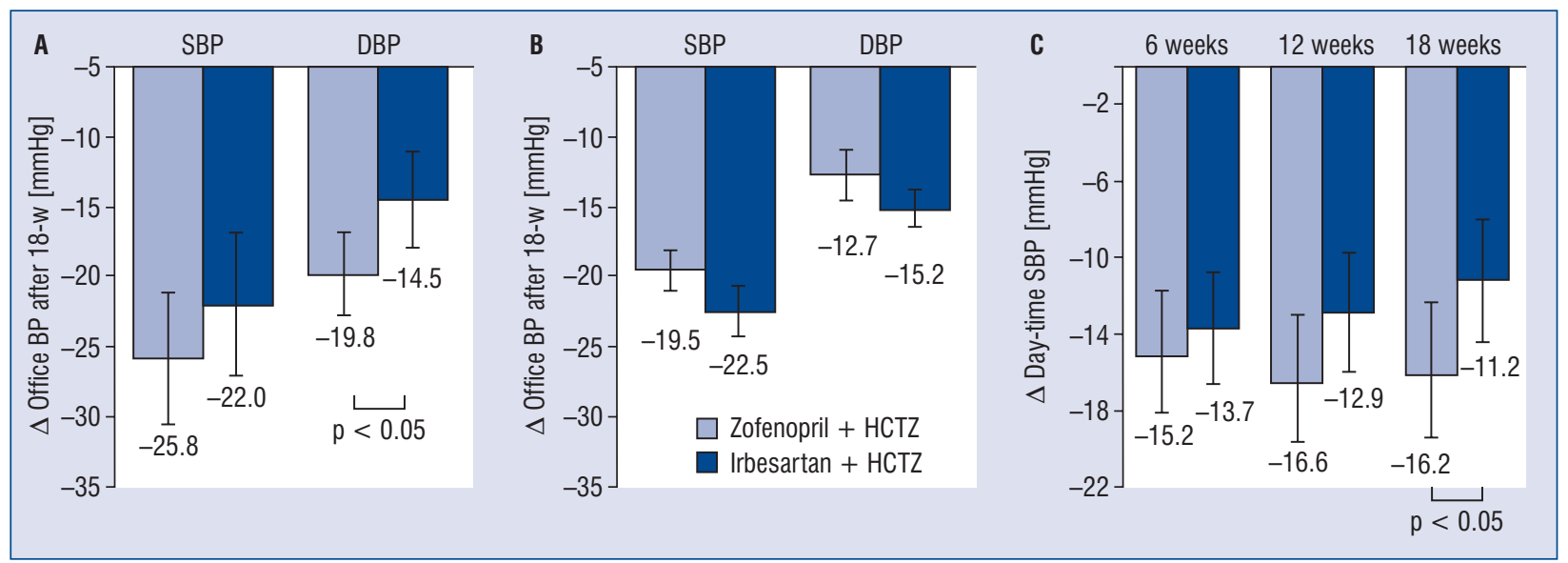

Figure 3. Mean changes $(\Delta)$ with treatment (and 95\% confidence interval) in office systolic blood pressure (SBP) and diastolic blood pressure (DBP) in the ZODIAC $(n=124)(A)$ and ZENITH study $(n=223)(B)$, and mean daytime SBP changes in the ZEUS study $(n=77)(C)$, in the subgroup of patients receiving the low drug doses during the study. The $\mathrm{p}$ values refer to the statistical significance of the between-treatment difference (reproduced from [33]); BP — blood pressure; HCTZ — hydrochlorothiazide.

results of the ZEUS study indicate that 18 weeks of treatment with both zofenopril 30-60 mg + HCTZ $12.5 \mathrm{mg}$ and irbesartan 150-300 $\mathrm{mg}+\mathrm{HCTZ} 12.5 \mathrm{mg}$ was effective in reducing and controlling daytime SBP in elderly patients with ISH. However, when maintained at a low dose, long-term treatment with zofenopril plus the thiazide diuretic was superior to the irbesartan-based combination [32].

A pooled analysis of the results of the $Z$-studies $(n=1469)$ confirmed the efficacy of both combinations on office and ambulatory BPs after 18-24 weeks, with similar reductions over $24 \mathrm{~h}$ and over the last $6 \mathrm{~h}$, both with the high and low dose combinations [33]. The analysis of data from the low-dose subgroups (corresponding to the currently available doses on the market) showed a higher efficacy of zofenopril $30 \mathrm{mg}+\mathrm{HCTZ}$ $12.5 \mathrm{mg}$ vs. irbesartan $150 \mathrm{mg}+\mathrm{HCTZ} 12.5 \mathrm{mg}$ in the ZODIAC study in terms of office DBP reductions (19.8 vs. $14.5 \mathrm{mmHg}, \mathrm{p}=0.022$ ). Similarly, in the patients of the ZEUS study maintaining the low drug doses throughout the study, the magnitude of the daytime BP lowering was always slightly greater under zofenopril $30 \mathrm{mg}+\mathrm{HCTZ} 12.5 \mathrm{mg}$ than under irbesartan $150 \mathrm{mg}+\mathrm{HCTZ} 12.5 \mathrm{mg}$; in this study subgroup, a statistically significant $(p=0.028)$ difference in favor of zofenopril-treated patients was achieved at study end (16.2 vs. 11.2 $\mathrm{mmHg}$ ) (Fig. 3). For the low-dose subgroup, also the percentage of patients showing daytime SBP normalization $(<135 \mathrm{mmHg}$ ) and response (SBP $<135 \mathrm{mmHg}$ or reduction $>10 \mathrm{mmHg}$ ) at study end was significantly larger under zofenopril (88.9\% and $91.7 \%)$ than under irbesartan $(73.2 \%$ and $78.0 \%$; $\mathrm{p}=0.017$ and $\mathrm{p}=0.024$, respectively) [33].

As far as the safety profile is concerned, in the $Z$-studies the total number of adverse events, as well as the proportion of treatment-related adverse events, was limited and similar between the two treatments $(25.2 \%$ of patients receiving zofenopril and $21.9 \%$ of patients receiving irbesartan; $\mathrm{p}=0.715$ ) [33]. This is consistent with the safety profile of the zofenopril + HCTZ combination derived from clinical trials, in which it was associated with a low percentage of treatment withdrawals [26]. Similarly, in the Z-studies, only 66 (4.3\%) patients were withdrawn from the studies because of an adverse event -38 in the zofenopril $(4.9 \%)$ and 28 in the irbesartan treatment group $(3.6 \% ; p=0.593)$. The most common drug-related adverse event observed under zofenopril was cough (1.8\% of patients), whereas dizziness was the most prevalent drug adverse reaction in irbesartan-treated patients (1.4\%) [33].

All these results support the usefulness of zofenopril + HCTZ fixed dose combination in the treatment of high-risk hypertensive patients or patients not adequately controlled by monotherapy: a further advantage of zofenopril in these patients is the lack of interaction with ASA, which may reduce the benefit of treatment with ACEIs in patients with chronic coronary heart disease, including those with HF [34]. This is of particular interest in complicated patients because antiplatelet therapy, in particular low-dose ASA, is recom- 
mended for secondary prevention in hypertensive patients [1].

\section{Treatment adherence}

Medication adherence is a growing concern to clinicians and healthcare systems because of mounting evidence that nonadherence is very frequent and associated with adverse outcomes and higher costs of care. Nonadherence to medications is common for patients with CVDs; in particular, about half of all patients prescribed antihypertensive medications have been found to stop taking them within 1 year of the initial prescription [35], with an average non-adherence rate in patients with resistant HTN of $31.2 \%$ [36]. This has a negative impact on treatment effectiveness because high adherence (defined as medication possession ratio of $80 \%$ to $100 \%$ ) to antihypertensive medications has been shown to be associated with a higher probability of BP control compared with medium or low levels of adherence [35].

The reasons for non-adherence belong to different categories, many of which apply to HTN; the World Health Organization has categorized potential reasons for medication nonadherence into 5 broad groupings that include patient-, condition-, therapy-, socioeconomic-, and health system-related factors [35]. For therapy-related factors, the complexity of the regimen and the perceived or experienced side effects can impact adherence [35].

Side effects are a big problem in anti-hypertensive treatment: a meta-analysis of 354 randomized double-blind placebo controlled HTN trials including 40,000 treated patients and 16,000 patients given placebo showed that most anti-hypertensive classes (except ACEIs) are associated with dose-related side effects, which might reduce adherence [37]. The choice of antihypertensive regimen should therefore be based on a careful evaluation of both the efficacy and tolerability profile of available drugs, in order to minimize side effects and maximize adherence, thus optimizing treatment efficacy.

Combinations of 2 antihypertensive drugs at fixed doses in a single tablet may be recommended and favored in chronic conditions like HTN, because reducing the number of daily pills improves adherence: non-compliance to medication regimens is reduced by $24-26 \%$ with fixed-dose combination regimens, which provide a strong armamentarium in chronic disease management [38]. Simplifying therapeutic regimens by using effective and well tolerated fixed dose combinations is therefore a successful strategy to improve adherence to antihypertensive therapy: the combination of medications targeting multiple mechanisms reduces the heterogeneity of the BP response to initial treatment and provides a steeper dose response than with escalating doses of monotherapy [1]. Moreover, whereas reductions in BP with drugs in combination are additive, adverse effects are less than additive [37].

\section{Conclusions}

Angiotensin converting enzyme inhibitors are a heterogeneous class of CV drugs, exerting beneficial effects in patients with, or at risk of, CVD. Differences in the pharmacological profile of ACEIs can significantly impact clinical efficacy. sulfhydryl-donor ACEIs are characterized by important ancillary properties (greater tissue selectivity, anti-oxidant effect, direct and indirect increase of NO availability). Zofenopril is an ACEI with unique properties due to its sulfhydryl group (high lipophilicity, free radical scavenging properties, and selective cardiac ACE inhibition, cardioprotective activity), which can be of particular value in patients with coronary artery disease. The positive impact of the cardio-protective effect of zofenopril on clinical outcomes has been extensively documented by the SMILE project, including several clinical trials in patients with different conditions of myocardial ischemia treated with zofenopril. On the other hand, the results of $Z$-studies suggest that the fixed combination of zofenopril and HCTZ, thanks to its ancillary features (anti-inflammatory action, regression of organ damage, metabolic neutrality) and good tolerability profile, may have a relevant place in the treatment of high-risk or monotherapytreated, uncontrolled hypertensive patients requiring a more prompt, intensive and sustained $\mathrm{BP}$ reduction, in line with the recommendations of current guidelines (Central illustration). A "patient-oriented" integration of guidelines, based on a careful consideration of the differences across antihypertensive drugs, patient's characteristics, approach to treatment, and additional risk factors, is a mandatory strategy for the future treatment of HTN, taking into consideration the complexity of the condition beyond $\mathrm{BP}$.

\section{Acknowledgments}

Editorial assistance was provided by Content Ed Net. 


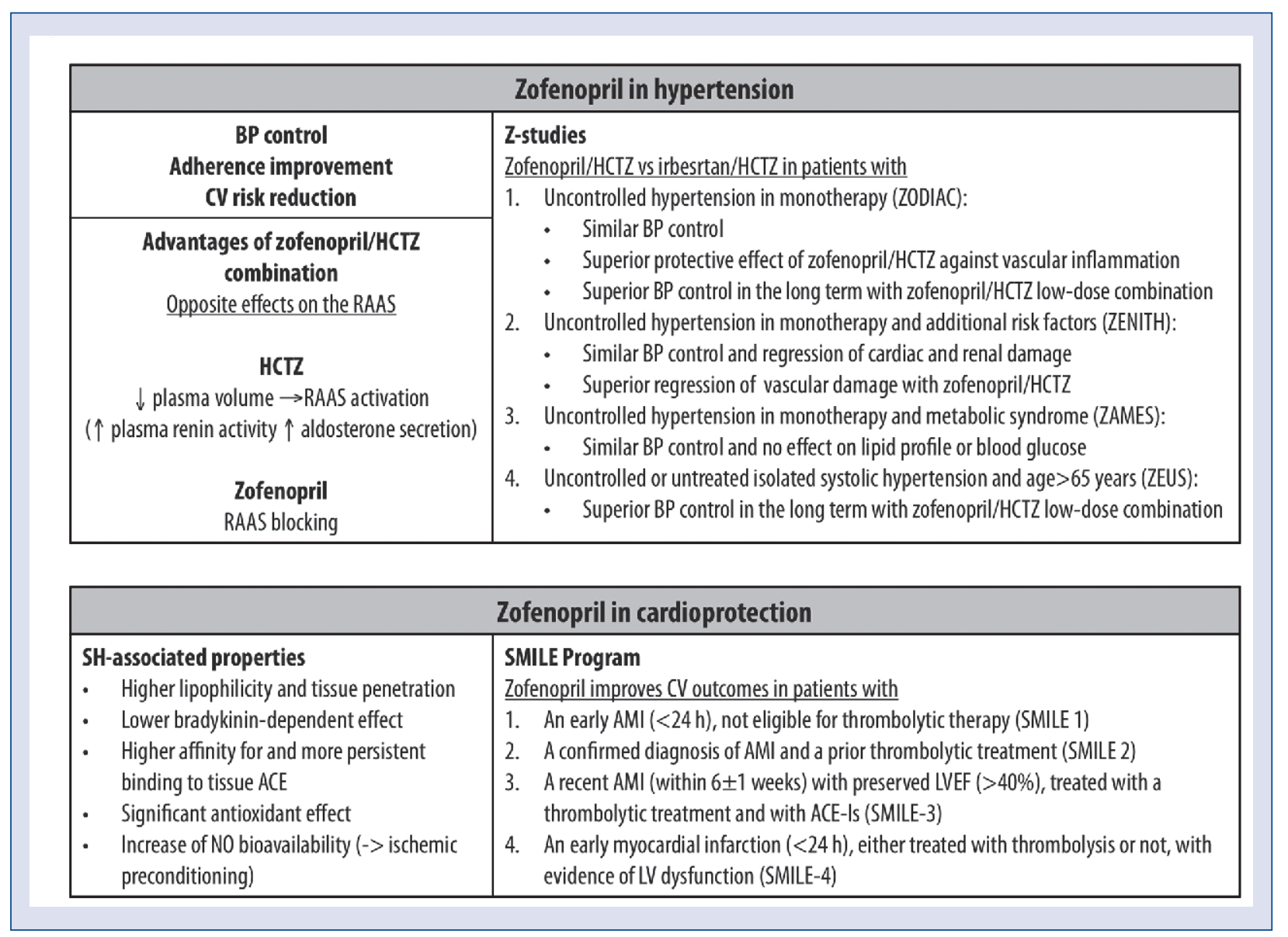

Central illustration. The role of zofenopril in blood pressure control and cardioprotection; ACEI — angiotensin converting enzyme inhibitors; AMI — acute myocardial infarction; BP — blood pressure; CV — cardiovascular; HCTZ — hydrochlorothiazide; LV — left ventricular; LVEF — left ventricular ejection fraction; NO — nitric oxide; RAAS — renin-angiotensin-aldosterone system; $\mathrm{SH}$ - sulfhydryl.

\section{Funding}

Financial support for editorial activity was supported by Menarini International Operations Luxemburg S.A.

Conflict of interest: Prof. Borghi received research grants from Menarini and honoraria as consultant or speaker from Alfasgma, Gilead, Menarini, Merck, Novartis, and Servier; Prof. Ambrosio received honoraria as a consultant or speaker from Bayer, Behring, Menarini, Merck, and Novartis; Prof. Van De Borne received honoraria as a consultant or speaker from Bayer, Boehringer-Ingelheim, Idorsia, Menarini, and Novartis; Prof. Mancia received honoraria for participation as a speaker/chairman in national or international meetings from AstraZeneca, Daiichi Sankyo, Medtronic, Menarini, Merck, Novartis, Recordati, Sanofi, and Servier.

\section{References}

1. Williams B, Mancia G, Spiering W, et al. 2018 ESC/ESH Guidelines for the management of arterial hypertension [published correction appears in Eur Heart J. 2019 Feb 1;40(5):475]. Eur Heart J. 2018; 39(33): 3021-3104.

2. Ambrosioni E. Defining the role of zofenopril in the management of hypertension and ischemic heart disorders. Am J Cardiovasc Drugs. 2007; 7(1): 17-24, doi: 10.2165/00129784-20070701000002, indexed in Pubmed: 17355163.

3. Mancia G, Fagard R, Narkiewicz K, et al. 2013 ESH/ESC Guidelines for the management of arterial hypertension. J Hypertens. 2013; 31(7): 1281-1357, doi: 10.1097/01.hjh.0000431740.32696.cc.

4. Borghi C, Omboni S, Reggiardo G, et al. Cardioprotective role of zofenopril in patients with acute myocardial infarction: a pooled individual data analysis of four randomised, doubleblind, controlled, prospective studies. Open Heart. 2015; 2(1): e000220, doi: 10.1136/openhrt-2014-000220, indexed in Pubmed: 26380097.

5. O'Gara P, Kushner F, Ascheim D, et al. 2013 ACCF/AHA guideline for the management of ST-elevation myocardial in- 
farction: a report of the American College of Cardiology Foundation/American Heart Association Task Force on Practice Guidelines [published correction appears in Circulation. 2013 Dec 24;128(25):e481]. Circulation. 2013; 127(4), doi: 10.1161/ cir.0b013e3182742cf6.

6. Task Force on the management of ST-segment elevation acute myocardial infarction of the European Society of Cardiology (ESC), Steg PG, James SK, et al. ESC Guidelines for the management of acute myocardial infarction in patients presenting with ST-segment elevation. Eur Heart J. 2012; 33(20): 2569-2619.

7. Subissi A, Evangelista S, Giachetti A. Preclinical profile of zofenopril: an angiotensin converting enzyme inhibitor with peculiar cardioprotective properties. Cardiovasc Drug Rev. 1999; 17(2): 115-133.

8. Cushman DW, Wang FL, Fung WC, et al. Differentiation of angiotensin-converting enzyme (ACE) inhibitors by their selective inhibition of ACE in physiologically important target organs. Am J Hypertens. 1989; 2(4): 294-306, doi: 10.1093/ajh/2.4.294, indexed in Pubmed: 2706094.

9. Ferrari R, Cargnoni A, Curello S, et al. Protection of the ischemic myocardium by the converting-enzyme inhibitor zofenopril: insight into its mechanism of action. J Cardiovasc Pharmacol. 1992; 20(5): 694-704, indexed in Pubmed: 1280730.

10. Donnarumma E, Ali MJ, Rushing AM, et al. Zofenopril protects against myocardial ischemia-reperfusion injury by increasing nitric oxide and hydrogen sulfide bioavailability. J Am Heart Assoc. 2016; 5(7), doi: 10.1161/JAHA.116.003531, indexed in Pubmed: 27381758.

11. Bucci M, Vellecco V, Cantalupo A, et al. Hydrogen sulfide accounts for the peripheral vascular effects of zofenopril independently of ACE inhibition. Cardiovasc Res. 2014; 102(1): 138-147, doi: 10.1093/cvr/cvu026, indexed in Pubmed: 24501330.

12. Scribner AW, Loscalzo J, Napoli C. The effect of angiotensinconverting enzyme inhibition on endothelial function and oxidant stress. Eur J Pharmacol. 2003; 482(1-3): 95-99, doi: 10.1016/j. ejphar.2003.10.002, indexed in Pubmed: 14660009.

13. Desideri G, Grassi D, Croce G, et al. Different effects of angiotensin converting enzyme inhibitors on endothelin-1 and nitric oxide balance in human vascular endothelial cells: evidence of an oxidant-sensitive pathway. Mediators Inflamm. 2008; 2008: 305087, doi: 10.1155/2008/305087, indexed in Pubmed: 19079593

14. Napoli C, Liguori A, Chiariello M, et al. New-onset angina preceding acute myocardial infarction is associated with improved contractile recovery after thrombolysis. Eur Heart J. 1998; 19(3): 411-419, doi: 10.1053/euhj.1997.0748, indexed in Pubmed: 9568445.

15. Ambrosio G, Del Pinto M, Tritto I, et al. Chronic nitrate therapy is associated with different presentation and evolution of acute coronary syndromes: insights from 52,693 patients in the Global Registry of Acute Coronary Events. Eur Heart J. 2010; 31(4): 430-438, doi: 10.1093/eurheartj/ehp457, indexed in Pubmed: 19903682

16. Cohen MV, Yang XM, Downey JM. Nitric oxide is a preconditioning mimetic and cardioprotectant and is the basis of many available infarct-sparing strategies. Cardiovasc Res. 2006; 70(2): 231-239, doi: 10.1016/j.cardiores.2005.10.021, indexed in Pubmed: 16376869 .

17. Ambrosioni E, Borghi C, Magnani B. Early treatment of acute myocardial infarction with angiotensin-converting enzyme inhibition: safety considerations. SMILE pilot study working party.
Am J Cardiol. 1991; 68(14): 101D-110D, doi: 10.1016/00029149(91)90266-n, indexed in Pubmed: 1746414.

18. Ambrosioni E, Borghi C, Magnani B. The effect of the angiotensin-converting-enzyme inhibitor zofenopril on mortality and morbidity after anterior myocardial infarction. The Survival of Myocardial Infarction Long-Term Evaluation (SMILE) Study Investigators. N Engl J Med. 1995; 332(2): 80-85, doi: 10.1056/ NEJM199501123320203, indexed in Pubmed: 7990904.

19. Borghi C, Ambrosioni E. Survival of Myocardial Infarction Longterm Evaluation-2 Working Party. Double-blind comparison between zofenopril and lisinopril in patients with acute myocardial infarction: results of the Survival of Myocardial Infarction Longterm Evaluation-2 (SMILE-2) study. Am Heart J. 2003; 145(1): 80-87, doi: 10.1067/mhj.2003.24, indexed in Pubmed: 12514658.

20. Borghi C, Ambrosioni E. Survival of Myocardial Infarction Longterm Evaluation Study Group. Effects of zofenopril on myocardial ischemia in post-myocardial infarction patients with preserved left ventricular function: the Survival of Myocardial Infarction Long-term Evaluation (SMILE)-ISCHEMIA study. Am Heart J. 2007; 153(3): 445.e7-445.14, doi: 10.1016/j.ahj.2006.12.005, indexed in Pubmed: 17307427.

21. Borghi C, Ambrosioni E, Novo S, et al. SMILE-4 Working Party. Comparison between zofenopril and ramipril in combination with acetylsalicylic acid in patients with left ventricular systolic dysfunction after acute myocardial infarction: results of a randomized, double-blind, parallel-group, multicenter, European study (SMILE-4). Clin Cardiol. 2012; 35(7): 416-423, doi: 10.1002/clc.22017, indexed in Pubmed: 22707187.

22. Borghi C, Ambrosioni E, Omboni S, et al. SMILE-4 Working Party. Zofenopril and ramipril and acetylsalicylic acid in postmyocardial infarction patients with left ventricular systolic dysfunction: a retrospective analysis in hypertensive patients of the SMILE-4 study. J Hypertens. 2013; 31(6): 1256-1264, doi: 10.1097/HJH.0b013e3283605cd8, indexed in Pubmed: 23552127.

23. Borghi C, Omboni S, Novo S, et al. Early Treatment With Zofenopril and Ramipril in Combination With Acetyl Salicylic Acid in Patients With Left Ventricular Systolic Dysfunction After Acute Myocardial Infarction: Results of a 5-Year Follow-up of Patients of the SMILE-4 Study. J Cardiovasc Pharmacol. 2017; 69(5): 298-304, doi: 10.1097/ FJC.0000000000000473, indexed in Pubmed: 28195948.

24. Borghi C, Omboni S. Zofenopril plus hydrochlorothiazide combination in the treatment of hypertension: an update. Expert Rev Cardiovasc Ther. 2014; 12(9): 1055-1065, doi: 10.1586/14779072.2014.946405, indexed in Pubmed: 25098297.

25. Parati G, Omboni S, Malacco E, et al. Antihypertensive efficacy of zofenopril and hydrochlorothiazide combination on ambulatory blood pressure. Blood Pressure. 2009; 15(sup1): 7-17, doi: 10.1080/08038020500501975.

26. Zanchetti A, Parati G, Malacco E. Zofenopril plus hydrochlorothiazide: Combination therapy for the treatment of mild to moderate hypertension. Drugs. 2006; 66(8): 1107-1115, doi: 10.2165/00003495200666080-00006, indexed in Pubmed: 16789795.

27. Malacco E, Omboni S. Antihypertensive efficacy of zofenopril plus hydrochlorothiazide fixed combination for treatment in metabolic syndrome. Adv Ther. 2007; 24(5): 1006-1015, doi: 10.1007/BF02877705, indexed in Pubmed: 18029326.

28. Omboni S, Malacco E, Parati G. Zofenopril plus hydrochlorothiazide fixed combination in the treatment of hypertension and associated clinical conditions. Cardiovasc Ther. 2009; 27(4): 275-288, doi: 10.1111/j.1755-5922.2009.00102.x, indexed in Pubmed: 19832845. 
29. Agabiti-Rosei E, Manolis A, Zava D, et al. ZODIAC Study Group. Zofenopril plus hydrochlorothiazide and irbesartan plus hydrochlorothiazide in previously treated and uncontrolled diabetic and non-diabetic essential hypertensive patients. Adv Ther. 2014; 31(2): 217-233, doi: 10.1007/s12325-013-0090-8, indexed in Pubmed: 24415271.

30. Malacco E, Omboni S, Parati G. Blood Pressure Response to Zofenopril or Irbesartan Each Combined with Hydrochlorothiazide in High-Risk Hypertensives Uncontrolled by Monotherapy: A Randomized, Double-Blind, Controlled, Parallel Group, Noninferiority Trial. Int J Hypertens. 2015; 2015: 139465, doi: 10.1155/2015/139465, indexed in Pubmed: 26347187.

31. Napoli C, Omboni S, Borghi C, et al. ZAMES (Zofenopril in Advanced MEtabolic Syndrome) Study Group. Fixed-dose combination of zofenopril plus hydrochlorothiazide vs. irbesartan plus hydrochlorothiazide in hypertensive patients with established metabolic syndrome uncontrolled by previous monotherapy. The ZAMES study (Zofenopril in Advanced MEtabolic Syndrome). J Hypertens. 2016; 34(11): 2287-2297, doi: 10.1097/ HJH.0000000000001079, indexed in Pubmed: 27653164.

32. Modesti P, Omboni S, Taddei S, et al. Zofenopril or irbesartan plus hydrochlorothiazide in elderly patients with isolated systolic hypertension untreated or uncontrolled by previous treatment. J Hypertens. 2016; 34(3): 567-587, doi: 10.1097/ hjh.0000000000000805.

33. Omboni S, Malacco E, Napoli C, et al. Erratum to: Efficacy of Zofenopril vs. Irbesartan in Combination with a Thiazide Diuretic in Hypertensive Patients with Multiple Risk Factors not Controlled by a Previous Monotherapy: A Review of the Double-Blind, Randomized „Z” Studies. Adv Ther. 2017; 34(6): 1498-1499, doi: 10.1007/s12325-017-0537-4, indexed in Pubmed: 28452039.

34. Jhund P, McMurray JJV. Does aspirin reduce the benefit of an angiotensin-converting enzyme inhibitor? Choosing between the Scylla of observational studies and the Charybdis of subgroup analysis. Circulation. 2006; 113(22): 2566-2568, doi: 10.1161/CIRCULATIONAHA.106.629212, indexed in Pubmed: 16754810.

35. Ho PM, Bryson CL, Rumsfeld JS. Medication adherence: its importance in cardiovascular outcomes. Circulation. 2009; 119(23): 3028-3035, doi: 10.1161/CIRCULATIONAHA.108.768986, indexed in Pubmed: 19528344.

36. Durand H, Hayes P, Morrissey EC, et al. Medication adherence among patients with apparent treatment-resistant hypertension: systematic review and meta-analysis. J Hypertens. 2017; 35(12): 2346-2357, doi: 10.1097/HJH.0000000000001502, indexed in Pubmed: 28777133.

37. Law MR, Wald NJ, Morris JK, et al. Value of low dose combination treatment with blood pressure lowering drugs: analysis of 354 randomised trials. BMJ. 2003; 326(7404): 1427, doi: 10.1136/ bmj.326.7404.1427, indexed in Pubmed: 12829555.

38. Bangalore S, Kamalakkannan G, Parkar S, et al. Fixed-dose combinations improve medication compliance: a meta-analysis. Am J Med. 2007; 120(8): 713-719, doi: 10.1016/j.amjmed.2006.08.033, indexed in Pubmed: 17679131. 